\title{
Fixed point and homotopy results for mixed multi-valued mappings in 0-complete partial metric spaces*
}

\author{
Maria Stella Adamo, Calogero Vetro \\ Department of Mathematics and Computer Sciences \\ University of Palermo \\ Via Archirafi 34, 90123 Palermo, Italy \\ calogero.vetro@unipa.it
}

Received: November 28, 2013 / Revised: April 23, 2014 / Published online: December 1, 2014

\begin{abstract}
We give sufficient conditions for the existence of common fixed points for a pair of mixed multi-valued mappings in the setting of 0-complete partial metric spaces. An example is given to demonstrate the usefulness of our results over the existing results in metric spaces. Finally, we prove a homotopy theorem via fixed point results.
\end{abstract}

Keywords: fixed points, multi-valued mappings, partial metric spaces.

\section{Introduction}

In the last century, the concept of metric space was largely studied and generalized in many directions. One of the most interesting is due to Matthews [17], which introduced the concept of partial metric as a part of the study of denotational semantics of dataflow networks. Several authors followed the ideas in [17] and proved many results, especially in fixed point theory; see $[1,4,5,7,10,11,15,16,19,22,25]$.

On the other hand, the study of multi-valued mappings received much attention in the last decades, because of its applications in mathematical optimization, control theory and differential inclusions [20]. We can say that this theory lies at the junction of topology, theory of functions and nonlinear functional analysis. In particular, we recall that Nadler [18] combined the concepts of contraction and multi-valued mapping by establishing the following fixed point result.

Theorem 1. (See [18].) Let $(X, d)$ be a complete metric space and $T: X \rightarrow C B(X)$ be a multi-valued mapping satisfying $H(T x, T y) \leqslant k d(x, y)$ for all $x, y \in X$, where $k$ is a constant such that $k \in(0,1)$ and $C B(X)$ denotes the family of non-empty closed and bounded subsets of $X$. Then $T$ has a fixed point, that is, there exists a point $u \in X$ such that $u \in T u$.

* This research was supported by the University of Palermo, Local University Project R.S. ex 60\%. 
Later on, a variety of generalizations, extensions and applications of this result appeared in the literature $[9,12,13,14,21,23]$. In particular, Aydi et al. [6] introduced the concept of partial Hausdorff metric and extended Theorem 1 in the setting of partial metric spaces.

Very recently, Aleomraninejad et al. [2] discussed the existence of fixed points for multi-valued mappings in the classical setting of metric spaces. Precisely, they proved fixed point theorems, which generalize known results in the literature, by using a suitable continuous function. These results were generalized by Yingtaweesittikul [26] to the setting of $b$-metric spaces. In view of the above considerations, we investigate the possibility to extend the results in $[2,26]$ to the setting of partial Hausdorff metric spaces. Also, our theorem and corollaries generalize and complement well known results in the literature on partial metric spaces. An example is given to demonstrate the usefulness of our results over the existing results in metric spaces. Finally, we prove a homotopy theorem via fixed point results.

\section{Preliminaries}

The aim of this section is to give some definitions and known results needed in the sequel. Let $\mathbb{R}^{+}$be the set of all non-negative real numbers and $\mathbb{N}$ the set of all positive integers.

We start with some concepts related to partial metric spaces.

Definition 1. (See [17].) A partial metric on a non-empty set $X$ is a mapping $p: X \times$ $X \rightarrow \mathbb{R}^{+}$such that, for all $x, y, z \in X$, the following conditions are satisfied:

(P1) $x=y \Leftrightarrow p(x, x)=p(x, y)=p(y, y)$;

(P2) $p(x, x) \leqslant p(x, y)$;

(P3) $p(x, y)=p(y, x)$;

(P4) $p(x, y) \leqslant p(x, z)+p(z, y)-p(z, z)$.

A non-empty set $X$ equipped with a partial metric $p$ is called partial metric space. We shall denote it by a pair $(X, p)$.

If $p(x, y)=0$, then (P1) and (P2) imply that $x=y$, but the converse does not hold true always.

Notice that if $p$ is a partial metric on $X$, then the mapping $p^{s}: X \times X \rightarrow \mathbb{R}^{+}$given by

$$
p^{s}(x, y)=2 p(x, y)-p(x, x)-p(y, y)
$$

is a metric on $X$. Also, each partial metric $p$ on $X$ generates a $T_{0}$ topology $\gamma_{p}$ on $X$, which has as a base, the family of the open balls ( $p$-balls) $\left\{B_{p}(x, \varepsilon): x \in X, \varepsilon>0\right\}$, where

$$
B_{p}(x, \varepsilon)=\{y \in X: p(x, y)<p(x, x)+\varepsilon\}
$$

for all $x \in X$ and $\varepsilon>0$.

Furthermore, $\lim _{n \rightarrow+\infty} p^{s}\left(x_{n}, x\right)=0$ if and only if

$$
p(x, x)=\lim _{n \rightarrow+\infty} p\left(x_{n}, x\right)=\lim _{n, m \rightarrow+\infty} p\left(x_{n}, x_{m}\right) .
$$


Definition 2. (See $[3,17]$.) Let $(X, p)$ be a partial metric space. Then a sequence $\left\{x_{n}\right\}$ is called:

(i) convergent, with respect to $\gamma_{p}$, if there exists some $x$ in $X$ such that $p(x, x)=$ $\lim _{n \rightarrow+\infty} p\left(x, x_{n}\right)$;

(ii) Cauchy sequence if there exists (and is finite) $\lim _{n, m \rightarrow+\infty} p\left(x_{n}, x_{m}\right)$.

A partial metric space $(X, p)$ is said to be complete if every Cauchy sequence $\left\{x_{n}\right\}$ in $X$ converges, with respect to $\gamma_{p}$, to a point $x \in X$ such that $p(x, x)=$ $\lim _{n, m \rightarrow+\infty} p\left(x_{n}, x_{m}\right)$.

A sequence $\left\{x_{n}\right\}$ in $(X, p)$ is called 0 -Cauchy if $\lim _{n, m \rightarrow+\infty} p\left(x_{n}, x_{m}\right)=0$. Also, we say that $(X, p)$ is 0 -complete if every 0 -Cauchy sequence in $X$ converges, with respect to the partial metric $p$, to a point $x \in X$ such that $p(x, x)=0$.

Lemma 1. (See $[3,17]$.) Let $(X, p)$ be a partial metric space. Then:

(i) A sequence $\left\{x_{n}\right\}$ is a Cauchy sequence in $(X, p)$ if and only if it is a Cauchy sequence in the metric space $\left(X, p^{s}\right)$;

(ii) $(X, p)$ is complete if and only if the metric space $\left(X, p^{s}\right)$ is complete.

Let $C B^{p}(X)$ be the collection of all non-empty closed and bounded subsets of $X$ with respect to the partial metric $p$. Consistent with Aydi et al. [6], closedness is taken from $\left(X, \gamma_{p}\right)$. Moreover, boundedness is given as follows: $A$ is a bounded subset in $(X, p)$ if there exist $x_{0} \in X$ and $M \geqslant 0$ such that, for all $a \in A$, we have $a \in B_{p}\left(x_{0}, M\right)$, that is, $p\left(x_{0}, a\right)<p\left(x_{0}, x_{0}\right)+M$. Then, for $A, B \in C B^{p}(X), x \in X, \delta_{p}: C B^{p}(X) \times$ $C B^{p}(X) \rightarrow \mathbb{R}^{+}$define

$$
\begin{array}{ll}
p(x, A)=\inf \{p(x, a): a \in A\}, & \delta_{p}(A, B)=\sup \{p(a, B): a \in A\}, \\
p(A, B)=\inf \{p(x, y): x \in A, y \in B\}, & \delta_{p}(B, A)=\sup \{p(b, A): b \in B\}
\end{array}
$$

and

$$
H_{p}(A, B)=\max \left\{\delta_{p}(A, B), \delta_{p}(B, A)\right\} .
$$

It is easy to show that $p(x, A)=0$ implies that $p^{s}(x, A)=0$, where

$$
p^{s}(x, A)=\inf \left\{p^{s}(x, a): a \in A\right\} .
$$

Proposition 1. (See [6].) Let $(X, p)$ be a partial metric space. For all $A, B, C \in C B^{p}(X)$, we have the following:

(i) $\delta_{p}(A, A)=\sup \{p(a, a): a \in A\}$;

(ii) $\delta_{p}(A, A) \leqslant \delta_{p}(A, B)$;

(iii) $\delta_{p}(A, B)=0$ implies that $A \subseteq B$;

(iv) $\delta_{p}(A, B) \leqslant \delta_{p}(A, C)+\delta_{p}(C, B)-\inf _{c \in C} p(c, c)$.

Proposition 2. (See [6].) Let $(X, p)$ be a partial metric space. For all $A, B, C \in C B^{p}(X)$, we have the following: 
(H1) $H_{p}(A, A) \leqslant H_{p}(A, B)$;

(H2) $H_{p}(A, B)=H_{p}(B, A)$;

(H3) $H_{p}(A, B) \leqslant H_{p}(A, C)+H_{p}(C, B)-\inf _{c \in C} p(c, c)$;

(H4) $H_{p}(A, B)=0 \Rightarrow A=B$.

The mapping $H_{p}: C B^{p}(X) \times C B^{p}(X) \rightarrow \mathbb{R}^{+}$is called the partial Hausdorff metric induced by $p$. Every Hausdorff metric is a partial Hausdorff metric but the converse is not true, see Example 2.6 in [6]. Also, a partial Hausdorff metric is not a partial metric, in general.

Lemma 2. (See [3].) Let $(X, p)$ be a partial metric space and A any non-empty set in $(X, p)$, then

$$
a \in \bar{A} \quad \Longleftrightarrow \quad p(a, A)=p(a, a),
$$

where $\bar{A}$ denotes the closure of $A$ with respect to the partial metric $p$. Notice that $A$ is closed in $(X, p)$ if and only if $A=\bar{A}$.

Lemma 3. (See [6].) Let (X,p) be a partial metric space, $A, B \in C B^{p}(X)$ and $h>1$, then, for any $a \in A$, there exists $b(a) \in B$ such that $p(a, b(a)) \leqslant h H_{p}(A, B)$.

Theorem 2. (See [6].) Let $(X, p)$ be a partial metric space. If $T: X \rightarrow C B^{p}(X)$ is a multi-valued mapping such that, for all $x, y \in X$, we have $H_{p}(T x, T y) \leqslant k p(x, y)$, where $k \in(0,1)$, then $T$ has a fixed point, that is, there exists a point $u \in X$ such that $u \in T u$.

Lemma 4. Let $(X, p)$ be a partial metric space and $T: X \rightarrow C B^{p}(X)$ a multi-valued mapping. If $\left\{x_{n}\right\} \subset X$ is a sequence, $x_{n} \rightarrow u$ and $p(u, u)=0$, then

$$
\lim _{n \rightarrow+\infty} p\left(x_{n}, T u\right)=p(u, T u) .
$$

Remark 1. Notice that the proof of Lemma 4 is an immediate consequence of the fact that the inequality

$$
p(u, T u)-p\left(u, x_{n}\right) \leqslant p\left(x_{n}, T u\right) \leqslant p\left(x_{n}, u\right)+p(u, T u)
$$

holds for all $n \in \mathbb{N}$.

Very recently, Romaguera [22] introduced the concept of mixed multi-valued mapping as follows.

Definition 3. (See [22].) Let $(X, p)$ be a partial metric space. $T: X \rightarrow X \cup C B^{p}(X)$ is called a mixed multi-valued mapping on $X$ if $T$ is a multi-valued mapping on $X$ such that, for each $x \in X,|T x|=1$ (i.e., $T x=\{y\}$ for some $y \in X$ ) or $T x \in C B^{p}(X)$.

A self-mapping $T: X \rightarrow X$ and a multi-valued mapping $T: X \rightarrow C B^{p}(X)$ are mixed multi-valued mappings.

According to [2] and [26], we consider a continuous function $g:\left(\mathbb{R}^{+}\right)^{5} \rightarrow \mathbb{R}^{+}$ satisfying the following conditions: 
1. $g(1,1,1,2,0)=g(1,1,1,0,2)=h \in(0,1)$;

2. $g$ is sub-homogeneous, that is, for all $\left(x_{1}, x_{2}, x_{3}, x_{4}, x_{5}\right) \in\left(\mathbb{R}^{+}\right)^{5}, \alpha>0$, we have

$$
g\left(\alpha x_{1}, \alpha x_{2}, \alpha x_{3}, \alpha x_{4}, \alpha x_{5}\right) \leqslant \alpha g\left(x_{1}, x_{2}, x_{3}, x_{4}, x_{5}\right) ;
$$

3. $g$ is a nondecreasing function, that is, for $x_{i}, y_{i} \in \mathbb{R}^{+}, x_{i} \leqslant y_{i}, i=1, \ldots, 5$, we have

$$
g\left(x_{1}, x_{2}, x_{3}, x_{4}, x_{5}\right) \leqslant g\left(y_{1}, y_{2}, y_{3}, y_{4}, y_{5}\right)
$$

and if $x_{i}, y_{i} \in \mathbb{R}^{+}, x_{i}<y_{i}$ for $i=1, \ldots, 4$, then

$$
\begin{aligned}
& g\left(x_{1}, x_{2}, x_{3}, x_{4}, 0\right)<g\left(y_{1}, y_{2}, y_{3}, y_{4}, 0\right), \\
& g\left(x_{1}, x_{2}, x_{3}, 0, x_{4}\right)<g\left(y_{1}, y_{2}, y_{3}, 0, y_{4}\right) .
\end{aligned}
$$

In this case, we write $g \in \mathcal{P}$; for results involving similar functions in metric spaces, see $[8]$ and the references therein.

Then we have the following lemma.

Lemma 5. If $g \in \mathcal{P}$ and $u, v \in \mathbb{R}^{+}$are such that

$$
\begin{gathered}
u \leqslant \max \{g(v, v, u, v+u, 0), g(v, v, u, 0, v+u), \\
g(v, u, v, v+u, 0), g(v, u, v, 0, v+u)\},
\end{gathered}
$$

then $u \leqslant h v$.

Proof. Without loss of generality, we can suppose that $u \leqslant g(v, v, u, v+u, 0)$. If $v \leqslant u$, then

$$
u \leqslant g(v, v, u, v+u, 0) \leqslant g(u, u, u, 2 u, 0) \leqslant u g(1,1,1,2,0)=h u<u,
$$

which is a contradiction. Thus, $u<v$ and

$$
u \leqslant g(v, v, u, v+u, 0) \leqslant g(v, v, v, 2 v, 0) \leqslant v g(1,1,1,2,0)=h v .
$$

\section{Main results}

Inspired by [2] and [26], we give the following results.

Lemma 6. Let $(X, p)$ be a partial metric space and let $F, G: X \rightarrow X \cup C B^{p}(X)$ be two mixed multi-valued mappings. Suppose that there exist $\alpha \in(0,1)$ and $g \in \mathcal{P}$ such that

implies that

$$
\min \{\alpha p(x, F x), \alpha p(y, G y)\} \leqslant p(x, y)
$$

$$
\begin{aligned}
H_{p}(F x, G y) \leqslant & g(p(x, y), p(x, F x), p(y, G y), p(x, G y)-p(x, x), \\
& p(y, F x)-p(y, y))
\end{aligned}
$$

for all $x, y \in X$. Then $\operatorname{Fix}(F)=\operatorname{Fix}(G)$. 
Proof. Let $x \in \operatorname{Fix}(F)$, then $p(x, F x)=p(x, x)$ and $\alpha p(x, F x)<p(x, x)$. Thus, we have

$$
\begin{aligned}
p(x, G x) & \leqslant H_{p}(F x, G x) \\
& \leqslant g(p(x, x), p(x, F x), p(x, G x), p(x, G x)-p(x, x), 0) \\
& \leqslant g(p(x, x), p(x, x), p(x, G x), p(x, G x)+p(x, x), 0) .
\end{aligned}
$$

Using Lemma 5, we get $p(x, G x) \leqslant h p(x, x) \leqslant p(x, x)$. On the other hand, by (P2) of Definition 1, we have $p(x, x) \leqslant p(x, G x)$ and so $p(x, x)=p(x, G x)$. Since $G x$ is a closed set, we conclude that $x \in G x$. Thus, $\operatorname{Fix}(F) \subseteq \operatorname{Fix}(G)$. Similarly, we deduce that $\operatorname{Fix}(G) \subseteq \operatorname{Fix}(F)$. This completes the proof.

Theorem 3. Let $(X, p)$ be a 0 -complete partial metric space and let $F, G: X \rightarrow X \cup$ $C B^{p}(X)$ be two mixed multi-valued mappings. Suppose that there exist $\alpha \in(0,1)$ and $g \in \mathcal{P}$ such that $\alpha(h+1) \leqslant 1$ and $\min \{\alpha p(x, F x), \alpha p(y, G y)\} \leqslant p(x, y)$ imply that

$$
\begin{aligned}
H_{p}(F x, G y) \leqslant & g(p(x, y), p(x, F x), p(y, G y), p(x, G y)-p(x, x), \\
& p(y, F x)-p(y, y))
\end{aligned}
$$

for all $x, y \in X$. Then $\operatorname{Fix}(F)=\operatorname{Fix}(G)$ and $\operatorname{Fix}(F)$ is a non-empty set.

Proof. By Lemma 6, $\operatorname{Fix}(F)=\operatorname{Fix}(G)$. Let $r \in(h, 1)$ and $x_{0} \in X$. If $x_{0}$ is not a fixed point, choose $x_{1} \in F x_{0}$, then $\alpha p\left(x_{0}, F x_{0}\right)<p\left(x_{0}, x_{1}\right)$. Consequently, we get

$$
\begin{aligned}
p\left(x_{1}, G x_{1}\right) \leqslant & H_{p}\left(F x_{0}, G x_{1}\right) \\
\leqslant & g\left(p\left(x_{0}, x_{1}\right), p\left(x_{0}, F x_{0}\right), p\left(x_{1}, G x_{1}\right), p\left(x_{0}, G x_{1}\right)-p\left(x_{0}, x_{0}\right),\right. \\
& \left.p\left(x_{1}, F x_{0}\right)-p\left(x_{1}, x_{1}\right)\right) \\
\leqslant & g\left(p\left(x_{0}, x_{1}\right), p\left(x_{0}, x_{1}\right), p\left(x_{1}, G x_{1}\right), p\left(x_{0}, x_{1}\right)+p\left(x_{1}, G x_{1}\right)\right. \\
& \left.-p\left(x_{0}, x_{0}\right)-p\left(x_{1}, x_{1}\right), 0\right) \\
\leqslant & g\left(p\left(x_{0}, x_{1}\right), p\left(x_{0}, x_{1}\right), p\left(x_{1}, G x_{1}\right), p\left(x_{0}, x_{1}\right)+p\left(x_{1}, G x_{1}\right), 0\right) .
\end{aligned}
$$

By Lemma 5, we have $p\left(x_{1}, G x_{1}\right) \leqslant h p\left(x_{0}, x_{1}\right)<r p\left(x_{0}, x_{1}\right)$. Now, if $x_{1}$ is not a fixed point, there exists $x_{2} \in G x_{1}$ such that $p\left(x_{1}, x_{2}\right)<r p\left(x_{0}, x_{1}\right)$. Since $\alpha p\left(x_{1}, G x_{1}\right)<$ $p\left(x_{1}, x_{2}\right)$, we have

$$
\begin{aligned}
p\left(x_{2}, F x_{2}\right) \leqslant & H_{p}\left(F x_{2}, G x_{1}\right) \\
\leqslant & g\left(p\left(x_{1}, x_{2}\right), p\left(x_{2}, F x_{2}\right), p\left(x_{1}, G x_{1}\right), p\left(x_{2}, G x_{1}\right)-p\left(x_{2}, x_{2}\right),\right. \\
& \left.p\left(x_{1}, F x_{2}\right)-p\left(x_{1}, x_{1}\right)\right) \\
\leqslant & g\left(p\left(x_{1}, x_{2}\right), p\left(x_{2}, F x_{2}\right), p\left(x_{1}, x_{2}\right), 0, p\left(x_{1}, x_{2}\right)+p\left(x_{2}, F x_{2}\right)\right. \\
& \left.-p\left(x_{1}, x_{1}\right)-p\left(x_{2}, x_{2}\right)\right) \\
\leqslant & g\left(p\left(x_{1}, x_{2}\right), p\left(x_{2}, F x_{2}\right), p\left(x_{1}, x_{2}\right), 0, p\left(x_{1}, x_{2}\right)+p\left(x_{2}, F x_{2}\right)\right) .
\end{aligned}
$$


By Lemma 5, we get $p\left(x_{2}, F x_{2}\right) \leqslant h p\left(x_{1}, x_{2}\right)<r p\left(x_{1}, x_{2}\right)$. Again, if $x_{2}$ is not a fixed point, there exists $x_{3} \in F x_{2}$ such that $p\left(x_{2}, x_{3}\right)<r p\left(x_{1}, x_{2}\right)<r^{2} p\left(x_{0}, x_{1}\right)$. Thus, by iterating this procedure, we can construct a sequence $\left\{x_{n}\right\}$ in $X$ satisfying

$$
\begin{gathered}
x_{2 n-1} \in F x_{2 n-2}, \quad x_{2 n} \in G x_{2 n-1}, \quad p\left(x_{n}, x_{n+1}\right)<r^{n} p\left(x_{0}, x_{1}\right), \\
p\left(x_{2 n}, F x_{2 n}\right) \leqslant h p\left(x_{2 n-1}, x_{2 n}\right), \quad p\left(x_{2 n-1}, G x_{2 n-1}\right) \leqslant h p\left(x_{2 n-2}, x_{2 n-1}\right) .
\end{gathered}
$$

The next step of the proof is to show that the sequence $\left\{x_{n}\right\}$ is a 0 -Cauchy sequence. Indeed, for each $q \in \mathbb{N}$, we have

$$
\begin{aligned}
& p\left(x_{n}, x_{n+q}\right) \\
& \leqslant p\left(x_{n}, x_{n+1}\right)+p\left(x_{n+1}, x_{n+q}\right)-p\left(x_{n+1}, x_{n+1}\right) \\
& \leqslant p\left(x_{n}, x_{n+1}\right)+p\left(x_{n+1}, x_{n+2}\right)+p\left(x_{n+2}, x_{n+q}\right)-p\left(x_{n+1}, x_{n+1}\right) \\
& \quad-p\left(x_{n+2}, x_{n+2}\right) \\
& \leqslant p\left(x_{n}, x_{n+1}\right)+p\left(x_{n+1}, x_{n+2}\right)+\cdots+p\left(x_{n+q-2}, x_{n+q-1}\right)+p\left(x_{n+q-1}, x_{n+q}\right) \\
& \quad \quad-\sum_{k=n+1}^{n+q-1} p\left(x_{k}, x_{k}\right) \\
& \leqslant \\
& \quad r^{n} p\left(x_{0}, x_{1}\right)+r^{n+1} p\left(x_{0}, x_{1}\right)+\cdots+r^{n+q-2} p\left(x_{0}, x_{1}\right)+r^{n+q-1} p\left(x_{0}, x\right) \\
& =r^{n} p\left(x_{0}, x_{1}\right)\left[1+r+r^{2}+\cdots+r^{q-1}\right] \\
& \leqslant
\end{aligned}
$$

Consequently, since

$$
\frac{r^{n}}{1-r} p\left(x_{0}, x_{1}\right) \rightarrow 0 \quad \text { as } n \rightarrow+\infty,
$$

we deduce that $\left\{x_{n}\right\}$ is a 0 -Cauchy sequence and so, by 0 -completeness of the space, $x_{n} \rightarrow x$ for some $x \in X$ with $p(x, x)=0$.

Now we claim that, for each $n \geqslant 1$, at least one of the following assertions holds:

$$
\alpha p\left(x_{2 n}, F x_{2 n}\right) \leqslant p\left(x_{2 n}, x\right) \quad \text { or } \quad \alpha p\left(x_{2 n+1}, G x_{2 n+1}\right) \leqslant p\left(x_{2 n+1}, x\right) .
$$

Suppose to the contrary that

$$
\alpha p\left(x_{2 n}, F x_{2 n}\right)>p\left(x_{2 n}, x\right) \quad \text { and } \quad \alpha p\left(x_{2 n+1}, G x_{2 n+1}\right)>p\left(x_{2 n+1}, x\right)
$$

for some $n \geqslant 1$, then we have

$$
\begin{aligned}
p\left(x_{2 n}, x_{2 n+1}\right) & \leqslant p\left(x_{2 n}, x\right)+p\left(x_{2 n+1}, x\right)-p(x, x) \\
& <\alpha\left[p\left(x_{2 n}, F x_{2 n}\right)+p\left(x_{2 n+1}, G x_{2 n+1}\right)\right] \\
& \leqslant \alpha\left[p\left(x_{2 n}, x_{2 n+1}\right)+h p\left(x_{2 n}, x_{2 n+1}\right)\right] \\
& =\alpha(h+1) p\left(x_{2 n}, x_{2 n+1}\right) .
\end{aligned}
$$


This leads to the contradiction $\alpha(h+1)>1$, and so the claim is proved. By using the assumption, for each $n \geqslant 1$ and $p(x, x)=0$, either

$$
\begin{aligned}
H_{p}\left(F x_{2 n}, G x\right) \leqslant & g\left(p\left(x_{2 n}, x\right), p\left(x_{2 n}, F x_{2 n}\right), p(x, G x),\right. \\
& \left.p\left(x_{2 n}, G x\right)-p\left(x_{2 n}, x_{2 n}\right), p\left(x, F x_{2 n}\right)\right)
\end{aligned}
$$

or

$$
\begin{aligned}
H_{p}\left(F x, G x_{2 n+1}\right) \leqslant & g\left(p\left(x, x_{2 n+1}\right), p(x, F x), p\left(x_{2 n+1}, G x_{2 n+1}\right),\right. \\
& \left.p\left(x, G x_{2 n+1}\right), p\left(x_{2 n+1}, F x\right)-p\left(x_{2 n+1}, x_{2 n+1}\right)\right)
\end{aligned}
$$

holds. Consequently, one of the following cases occurs:

(a) There exists an infinite subset $I \subseteq \mathbb{N}$ such that

$$
\begin{aligned}
p\left(x_{2 n+1}, G x\right) \leqslant & H_{p}\left(F x_{2 n}, G x\right) \\
\leqslant & g\left(p\left(x_{2 n}, x\right), p\left(x_{2 n}, F x_{2 n}\right), p(x, G x), p\left(x_{2 n}, G x\right)\right. \\
& \left.-p\left(x_{2 n}, x_{2 n}\right), p\left(x, F x_{2 n}\right)\right)
\end{aligned}
$$

for all $n \in I$;

(b) There exists an infinite subset $J \subseteq \mathbb{N}$ such that

$$
\begin{aligned}
p\left(F x, x_{2 n+2}\right) \leqslant & H_{p}\left(F x, G x_{2 n+1}\right) \\
\leqslant & g\left(p\left(x, x_{2 n+1}\right), p(x, F x), p\left(x_{2 n+1}, G x_{2 n+1}\right), p\left(x, G x_{2 n+1}\right),\right. \\
& \left.p\left(x_{2 n+1}, F x\right)-p\left(x_{2 n+1}, x_{2 n+1}\right)\right)
\end{aligned}
$$

for all $n \in J$.

Thus, in case $(a)$, we write

$$
\begin{aligned}
p(x, G x) \leqslant & p\left(x, x_{2 n+1}\right)+p\left(x_{2 n+1}, G x\right)-p\left(x_{2 n+1}, x_{2 n+1}\right) \\
\leqslant & p\left(x, x_{2 n+1}\right)-p\left(x_{2 n+1}, x_{2 n+1}\right)+g\left(p\left(x_{2 n}, x\right), p\left(x_{2 n}, F x_{2 n}\right), p(x, G x),\right. \\
& \left.p\left(x_{2 n}, G x\right)-p\left(x_{2 n}, x_{2 n}\right), p\left(x, F x_{2 n}\right)\right) \\
\leqslant & p\left(x, x_{2 n+1}\right)-p\left(x_{2 n+1}, x_{2 n+1}\right)+g\left(p\left(x_{2 n}, x\right), p\left(x_{2 n}, x_{2 n+1}\right), p(x, G x),\right. \\
& \left.p\left(x_{2 n}, x\right)+p(x, G x)-p\left(x_{2 n}, x_{2 n}\right), p\left(x, x_{2 n+1}\right)\right) \\
\leqslant & p\left(x, x_{2 n+1}\right)-p\left(x_{2 n+1}, x_{2 n+1}\right)+g\left(p\left(x_{2 n}, x\right), p\left(x_{2 n}, x_{2 n+1}\right), p(x, G x),\right. \\
& \left.p\left(x_{2 n}, x\right)+p(x, G x), p\left(x, x_{2 n+1}\right)\right)
\end{aligned}
$$

for all $n \in I$. Since $g$ is continuous, passing to limit as $n \rightarrow+\infty$, we obtain

$$
p(x, G x) \leqslant g(p(x, x), p(x, x), p(x, G x), p(x, x)+p(x, G x), 0) .
$$

This implies, by Lemma 5 , that $p(x, G x) \leqslant p(x, x)=0$ and so, by (P2) of Definition 1, we deduce that $p(x, G x)=p(x, x)=0$, that is, $x \in G x$ and hence, $\operatorname{Fix}(G)=$ $\operatorname{Fix}(F) \neq \emptyset$. 
On the other hand, in case $(b)$, we have

$$
\begin{aligned}
p(x, F x) \leqslant & p\left(x, x_{2 n+2}\right)+p\left(x_{2 n+2}, F x\right)-p\left(x_{2 n+2}, x_{2 n+2}\right) \\
\leqslant & p\left(x, x_{2 n+2}\right)-p\left(x_{2 n+2}, x_{2 n+2}\right)+g\left(p\left(x, x_{2 n+1}\right), p(x, F x),\right. \\
& \left.p\left(x_{2 n+1}, G x_{2 n+1}\right), p\left(x, G x_{2 n+1}\right), p\left(x_{2 n+1}, F x\right)-p\left(x_{2 n+1}, x_{2 n+1}\right)\right) \\
\leqslant & p\left(x, x_{2 n+2}\right)-p\left(x_{2 n+2}, x_{2 n+2}\right)+g\left(p\left(x, x_{2 n+1}\right), p(x, F x),\right. \\
& \left.p\left(x_{2 n+1}, x_{2 n+2}\right), p\left(x, x_{2 n+2}\right), p\left(x_{2 n+1}, x\right)+p(x, F x)-p\left(x_{2 n+1}, x_{2 n+1}\right)\right) \\
\leqslant & p\left(x, x_{2 n+2}\right)-p\left(x_{2 n+2}, x_{2 n+2}\right)+g\left(p\left(x, x_{2 n+1}\right), p(x, F x),\right. \\
& \left.p\left(x_{2 n+1}, x_{2 n+2}\right), p\left(x, x_{2 n+2}\right), p\left(x_{2 n+1}, x\right)+p(x, F x)\right)
\end{aligned}
$$

for all $n \in J$. Since $g$ is continuous, passing to limit as $n \rightarrow+\infty$, we obtain

$$
p(x, F x) \leqslant g(p(x, x), p(x, F x), p(x, x), 0, p(x, x)+p(x, F x)) .
$$

Also, by Lemma 5, we get $p(x, F x) \leqslant p(x, x)=0$ and so, by (P2) of Definition 1, we deduce that $p(x, F x)=p(x, x)$, that is, $x \in F x$. Thus, $\operatorname{Fix}(F) \neq \emptyset$. This completes the proof.

The following corollary is an immediate consequence of Theorem 3 in the case of a mixed multi-valued mapping.

Corollary 1. Let $(X, p)$ be a 0 -complete partial metric space and let $T: X \rightarrow X \cup$ $C B^{p}(X)$ be a mixed multi-valued mapping. Suppose that there exist $\alpha \in(0,1)$ and $g \in \mathcal{P}$ such that $\alpha(h+1) \leqslant 1$ and $\alpha p(x, T x) \leqslant p(x, y)$ imply that

$$
H_{p}(T x, T y) \leqslant g(p(x, y), p(x, T x), p(y, T y), p(x, T y)-p(x, x), p(y, T x)-p(y, y))
$$

for all $x, y \in X$. Then $T$ has a fixed point.

Moreover, we give some particular cases of Corollary 1, which can be used in applications.

Corollary 2. Let $(X, p)$ be a 0 -complete partial metric space and let $T: X \rightarrow X \cup$ $C B^{p}(X)$ be a mixed multi-valued mapping. Suppose that there exists $r \in(0,1)$ such that

$$
\begin{aligned}
& \frac{1}{r+1} p(x, T x) \leqslant p(x, y) \\
& \quad \Longrightarrow \quad H_{p}(T x, T y) \leqslant r \max \{p(x, y), p(x, T x), p(y, T y)\}
\end{aligned}
$$

for all $x, y \in X$. Then $T$ has a fixed point.

Proof. Let $g \in \mathcal{P}$ be defined by $g\left(x_{1}, x_{2}, x_{3}, x_{4}, x_{5}\right)=r \max \left\{x_{1}, x_{2}, x_{3}\right\}$, where $r \in$ $(0,1)$. Put $\alpha=1 /(r+1)$. Since $h=r<1$ and $\alpha(h+1) \leqslant 1$, by using Corollary 1 , we conclude that $T$ has a fixed point. 
Corollary 3. Let $(X, p)$ be a 0 -complete partial metric space and let $T: X \rightarrow X \cup$ $C B^{p}(X)$ be a mixed multi-valued mapping. Suppose that there exist $a, b, c \in[0,1)$ with $a+b+c<1$ such that

$$
\begin{aligned}
\frac{1}{1+a+b+c} p(x, T x) & \leqslant p(x, y) \\
\Longrightarrow \quad H_{p}(T x, T y) & \leqslant a p(x, y)+b p(x, T x)+c p(y, T y)
\end{aligned}
$$

for all $x, y \in X$. Then $T$ has a fixed point.

Proof. Let $g \in \mathcal{P}$ be defined by $g\left(x_{1}, x_{2}, x_{3}, x_{4}, x_{5}\right)=a x_{1}+b x_{2}+c x_{3}$, where $a+b+c<1$. Put $\alpha=1 /(a+b+c+1)$. Since $h=a+b+c<1$ and $\alpha(h+1) \leqslant 1$, by using Corollary 1, we conclude that $T$ has a fixed point.

Here we give an example, which illustrates the use of Corollary 2. Also, we show that this corollary is a valid generalization of the analogous result on metric spaces (see [2]).

Example 1. Consider the partial metric space $(X, p)$ with $X=\{0,1,2\}$ and $p: X \times X \rightarrow$ $\mathbb{R}^{+}$given by

$$
\begin{gathered}
p(0,0)=p(1,1)=0, \quad p(2,2)=\frac{1}{4}, \quad p(0,1)=p(1,0)=\frac{1}{3}, \\
p(0,2)=p(2,0)=\frac{2}{5}, \quad p(1,2)=p(2,1)=\frac{11}{15} .
\end{gathered}
$$

Clearly, $(X, p)$ is complete.

Also define $T: X \rightarrow C B^{p}(X)$ by

$$
T x= \begin{cases}\{0\} & \text { if } x \in\{0,1\} \\ \{0,1\} & \text { otherwise }\end{cases}
$$

Now we get

$$
\max \{p(x, T x): x \in X\}=\frac{2}{5} \quad \text { and } \quad \min \{p(x, y): x, y \in X, x \neq y\}=\frac{1}{3} .
$$

Note that, for each $r \in[1 / 5,1)$, we have

$$
\frac{1}{r+1} p(x, T x) \leqslant p(x, y)
$$

for all $x, y \in X$ with $x \neq y$. Putting $r=5 / 6$, we get

$$
\begin{aligned}
& H_{p}(T 0, T 1)=p(0,0)=0 \leqslant \frac{5}{6} \max \{p(0,1), p(0, T 0), p(1, T 1)\}, \\
& H_{p}(T 0, T 2)=p(0,1)=\frac{1}{3}=\frac{5}{6} p(0,2) \leqslant \frac{5}{6} \max \{p(0,2), p(0, T 0), p(2, T 2)\}, \\
& H_{p}(T 1, T 2)=p(0,1)=\frac{1}{3}<\frac{5}{6} p(1,2) \leqslant \frac{5}{6} \max \{p(1,2), p(1, T 1), p(2, T 2)\}
\end{aligned}
$$


and hence, for all $x, y \in X$ with $x \neq y$, condition (1) holds true. Also, condition (1) holds trivially for $x=y=0$, but is not applicable for $x, y \in\{1,2\}$ with $x=y$, since $1 /(r+1) p(x, T x) \nless p(x, x)$. Thus, all the conditions of Corollary 2 are satisfied and $x=0$ is a fixed point of $T$.

Next, we consider the metric space $\left(X, p^{s}\right)$, where the metric $p^{s}$, induced by the partial metric $p$, is given by

$$
\begin{gathered}
p^{s}(0,0)=p^{s}(1,1)=p^{s}(2,2)=0, \quad p^{s}(0,1)=p^{s}(1,0)=\frac{2}{3}, \\
p^{s}(1,2)=p^{s}(2,1)=\frac{73}{60}, \quad p^{s}(0,2)=p^{s}(2,0)=\frac{11}{20} .
\end{gathered}
$$

We show easily that Corollary 2.5 of [2] is not applicable in this case. Indeed, since

$$
\frac{1}{r+1} p^{s}(0, T 0)=\frac{1}{r+1} p^{s}(0,0)=0 \leqslant p^{s}(0, y)
$$

is satisfied for each $r \in(0,1)$ and $y \in X$, then, for $y=2$, we must have

$$
H_{p^{s}}(T 0, T 2) \leqslant r \max \left\{p^{s}(0,2), p^{s}(0, T 0), p^{s}(2, T 2)\right\} .
$$

After calculations, we get

and

$$
H_{p^{s}}(T 0, T 2)=H_{p^{s}}(\{0\},\{0,1\})=\frac{2}{3}
$$

$$
\max \left\{p^{s}(0,2), p^{s}(0, T 0), p^{s}(2, T 2)\right\}=\max \left\{\frac{11}{20}, 0, \frac{11}{20}\right\}=\frac{11}{20}<\frac{2}{3}
$$

Thus, for each $r \in(0,1)$, we have

$$
H_{p^{s}}(T 0, T 2) \nless r \max \left\{p^{s}(0,2), p^{s}(0, T 0), p^{s}(2, T 2)\right\}
$$

and so Corollary 2.5 of [2] does not hold with respect to the metric space $\left(X, p^{s}\right)$.

\section{Homotopy result in 0-complete partial metric spaces}

In this section, inspired by [24] and following a similar argument, we apply our Corollary 3 to get a homotopy result. Before establishing our theorem, we need the following proposition, which shows that if the multi-valued mapping $T: X \rightarrow C B^{p}(X)$ has a fixed point in $X$, then its self-distance is equal to zero.

Proposition 3. Let $(X, p)$ be a partial metric space and let $T: X \rightarrow C B^{p}(X)$ be a multivalued mapping satisfying (2). If $z \in T z$ for some $z \in X$, then $p(x, x)=0$ for all $x \in T z$, and hence, $H_{p}(T z, T z)=0$. 
Proof. Let $z \in T z \in C B^{p}(X)$ so that, by Lemma 2, $p(z, T z)=p(z, z)$ and $H_{p}(T z, T z)=$ $\delta_{p}(T z, T z)=\sup _{x \in T z} p(x, x)$. Consequently, assuming $p(z, z)>0$, by (2) we get

$$
\begin{gathered}
H_{p}(T z, T z) \leqslant a p(z, z)+b p(z, T z)+c p(z, T z), \\
\sup _{x \in T z} p(x, x) \leqslant(a+b+c) p(z, z), \quad \sup _{x \in T z} p(x, x)<p(z, z),
\end{gathered}
$$

which yields to contradiction, because $z \in T z$. This completes the proof.

Finally, we introduce the function $\eta:[\alpha, \beta]^{2} \rightarrow \mathbb{R}^{+}$such that one of the following conditions hold:

( $\eta 1)$ For all $r, s, t, u, v \in[\alpha, \beta]$, we have $\eta(t, r) \leqslant \eta(t, s)+\eta(r, s)$ and $\eta(u, v) \rightarrow 0$ if $u \rightarrow v$;

( $\eta 2)$ For all $s, t \in[\alpha, \beta]$ and some $L>0$, we have $\eta(t, s) \leqslant L|t-s|$.

Theorem 4. Let $(X, p)$ be a 0 -complete partial metric space, $F$ be a closed subset of $X$ and $U$ be a non-empty open subset of $X$ with $U \subset F$. Let $\alpha, \beta \in \mathbb{R}$ and $T: F \times[\alpha, \beta] \rightarrow$ $C B^{p}(X)$ be a multi-valued operator satisfying the following conditions:

(i) $x \notin T(x, t)$ for each $x \in F \backslash U$ and each $t \in[\alpha, \beta]$;

(ii) There exist $a, b, c \in[0,1)$ with $a+b+c<1$ and $b \leqslant c$ such that, for all $x, y \in F$ and each $t \in[\alpha, \beta]$,

implies that

$$
\frac{1}{1+a+b+c} p(x, T(x, t)) \leqslant p(x, y)
$$

$$
H_{p}(T(x, t), T(y, t)) \leqslant a p(x, y)+b p(x, T(x, t))+c p(y, T(y, t)) ;
$$

(iii) There exists $M>0$ such that, for all $t_{1}, t_{2} \in[\alpha, \beta]$ and each $x \in F$,

$$
H_{p}\left(T\left(x, t_{1}\right), T\left(x, t_{2}\right)\right) \leqslant M \eta\left(t_{1}, t_{2}\right) ;
$$

(iv) If $x \in T(x, t)$, then $T(x, t)=\{x\}$.

If $T\left(\cdot, t_{1}\right)$ has a fixed point in $F$ for at least one $t_{1} \in[\alpha, \beta]$, then $T(\cdot, t)$ has a fixed point in $U$ for all $t \in[\alpha, \beta]$. Furthermore, for any fixed $t \in[\alpha, \beta]$, the fixed point of $T(\cdot, t)$ is unique.

Proof. Define the set

$$
Q:=\{t \in[\alpha, \beta]: x \in T(x, t) \text { for some } x \in U\} .
$$

Since $T\left(\cdot, t_{1}\right)$ has a fixed point in $F$ for at least one $t_{1} \in[\alpha, \beta]$, that is, there exists $x \in F$ such that $x \in T\left(x, t_{1}\right)$ for at least one $t_{1} \in[\alpha, \beta]$ and (i) holds, therefore, $Q \neq \emptyset$. We shall show that $Q$ is both open and closed in $[\alpha, \beta]$ and so, by connectedness of $[\alpha, \beta]$, $Q=[\alpha, \beta]$. 
Step I: $Q$ is closed. Let $\left\{t_{n}\right\}$ be a sequence in $Q$ and $t_{n} \rightarrow s \in[\alpha, \beta]$ as $n \rightarrow+\infty$. We must show that $s \in Q$. Since $t_{n} \in Q$ for all $n \in \mathbb{N}$, there exists $x_{n} \in U$ with $x_{n} \in T\left(x_{n}, t_{n}\right)$ for all $n \in \mathbb{N}$. Now, for $n, m \in \mathbb{N}$ with $m>n$, using (ii), (iii) and Proposition 3, we obtain

$$
\begin{aligned}
H_{p}\left(T\left(x_{n}, t_{m}\right), T\left(x_{m}, t_{m}\right)\right) & \leqslant a p\left(x_{n}, x_{m}\right)+b p\left(x_{n}, T\left(x_{n}, t_{m}\right)\right)+c p\left(x_{m}, T\left(x_{m}, t_{m}\right)\right) \\
& \leqslant a p\left(x_{n}, x_{m}\right)+b H_{p}\left(T\left(x_{n}, t_{n}\right), T\left(x_{n}, t_{m}\right)\right),
\end{aligned}
$$

that is,

$$
H_{p}\left(T\left(x_{n}, t_{m}\right), T\left(x_{m}, t_{m}\right)\right) \leqslant a p\left(x_{n}, x_{m}\right)+b M \eta\left(t_{n}, t_{m}\right) .
$$

Thus, using the last inequality, (iv), and (H3) of Proposition 2, we get

$$
\begin{aligned}
p\left(x_{n}, x_{m}\right)= & H_{p}\left(T\left(x_{n}, t_{n}\right), T\left(x_{m}, t_{m}\right)\right) \\
\leqslant & H_{p}\left(T\left(x_{n}, t_{n}\right), T\left(x_{n}, t_{m}\right)\right)+H_{p}\left(T\left(x_{n}, t_{m}\right), T\left(x_{m}, t_{m}\right)\right) \\
& -\inf _{x \in T\left(x_{n}, t_{m}\right)} p(x, x) \\
\leqslant & H_{p}\left(T\left(x_{n}, t_{n}\right), T\left(x_{n}, t_{m}\right)\right)+H_{p}\left(T\left(x_{n}, t_{m}\right), T\left(x_{m}, t_{m}\right)\right) \\
\leqslant & M \eta\left(t_{n}, t_{m}\right)+a p\left(x_{n}, x_{m}\right)+b M \eta\left(t_{n}, t_{m}\right) \\
= & (1+b) M \eta\left(t_{n}, t_{m}\right)+a p\left(x_{n}, x_{m}\right) .
\end{aligned}
$$

Since $a<1$, we have

$$
p\left(x_{n}, x_{m}\right) \leqslant \frac{1+b}{1-a} M \eta\left(t_{n}, t_{m}\right) .
$$

From the properties of function $\eta$ and the fact that $t_{n} \rightarrow s$ as $n \rightarrow+\infty$, we deduce that $\eta\left(t_{n}, t_{m}\right) \rightarrow 0$ as $n \rightarrow+\infty$ and hence,

$$
\lim _{n \rightarrow+\infty} p\left(x_{n}, x_{m}\right)=0 .
$$

Therefore, the sequence $\left\{x_{n}\right\}$ is 0 -Cauchy in $F,(X, p)$ is 0 -complete and $F$ is closed. This implies that there exists $z \in F$ such that

$$
\lim _{n \rightarrow+\infty} p\left(x_{n}, z\right)=p(z, z)=0
$$

Moreover, for all $n \in \mathbb{N}$, we have

$$
p\left(x_{n}, T\left(x_{n}, s\right)\right) \leqslant H_{p}\left(T\left(x_{n}, t_{n}\right), T\left(x_{n}, s\right)\right) \leqslant M \eta\left(t_{n}, s\right)
$$

and hence, using again the properties of function $\eta$, it follows that

This implies

$$
\lim _{n \rightarrow+\infty} p\left(x_{n}, T\left(x_{n}, s\right)\right)=0 .
$$

$$
\lim _{n \rightarrow+\infty} p\left(z, T\left(x_{n}, s\right)\right) \leqslant \lim _{n \rightarrow+\infty}\left(p\left(z, x_{n}\right)+p\left(x_{n}, T\left(x_{n}, s\right)\right)\right)=0 .
$$


Next,

$$
\begin{aligned}
p\left(x_{n}, T(z, s)\right) \leqslant & H_{p}\left(T\left(x_{n}, t_{n}\right), T(z, s)\right) \\
\leqslant & H_{p}\left(T\left(x_{n}, t_{n}\right), T\left(x_{n}, s\right)\right)+H_{p}\left(T\left(x_{n}, s\right), T(z, s)\right) \\
& -\inf _{x \in T\left(x_{n}, s\right)} p(x, x) \\
\leqslant & M \eta\left(t_{n}, s\right)+a p\left(x_{n}, z\right)+b p\left(x_{n}, T\left(x_{n}, s\right)\right)+c p(z, T(z, s)),
\end{aligned}
$$

that is,

$$
p\left(x_{n}, T(z, s)\right) \leqslant M \eta\left(t_{n}, s\right)+a p\left(x_{n}, z\right)+b p\left(x_{n}, T\left(x_{n}, s\right)\right)+c p(z, T(z, s)) .
$$

From above, it follows easily that

$$
\begin{aligned}
p(z, T(z, s)) & \leqslant p\left(z, x_{n}\right)+p\left(x_{n}, T(z, s)\right) \\
& =p\left(z, x_{n}\right)+M \eta\left(t_{n}, s\right)+a p\left(x_{n}, z\right)+b p\left(x_{n}, T\left(x_{n}, s\right)\right)+c p(z, T(z, s)),
\end{aligned}
$$

that is,

$$
(1-c) p(z, T(z, s)) \leqslant(1+a) p\left(z, x_{n}\right)+M \eta\left(t_{n}, s\right)+b p\left(x_{n}, T\left(x_{n}, s\right)\right) .
$$

Since $p\left(x_{n}, T\left(x_{n}, s\right)\right) \rightarrow 0$ as $n \rightarrow+\infty, 1-c>0$ and using the properties of function $\eta$, from above inequality we deduce that $p(z, T(z, s))=0$. Therefore, $z \in T(z, s)$, and from (i) we obtain $z \in U$. Thus, $s \in Q$ and hence, $Q$ is closed in $[\alpha, \beta]$.

Step II: $Q$ is open. Let $t_{0} \in Q$ and $x_{0} \in U$ with $x_{0} \in T\left(x_{0}, t_{0}\right)$. Note that, for such $t_{0}$, Proposition 3 is applicable and hence,

$$
p\left(x_{0}, x_{0}\right)=0 .
$$

Since $U$ is open, there exists $r>0$ such that $B_{p}\left(x_{0}, r\right) \subset U$. Now, assume $\epsilon=$ $((1-k) / M) r>0$ with $k=(a+b) /(1-b)<1$. By definition of function $\eta$, we can choose $\delta>0$ such that $\eta\left(t, t_{0}\right)<\epsilon$ for all $t \in\left(t_{0}-\delta, t_{0}+\delta\right)$.

Let $t \in\left(t_{0}-\delta, t_{0}+\delta\right)$, then, for all $x \in \overline{B_{p}\left(x_{0}, r\right)}=\left\{x \in X: p\left(x, x_{0}\right) \leqslant r+\right.$ $\underline{\left.p\left(x_{0}, x_{0}\right)\right\}}=\left\{x \in X: p\left(\underline{\left.x, x_{0}\right) \leqslant r}\right\}\right.$ (as $\left.p\left(x_{0}, x_{0}\right)=0\right)$, we shall show that $T(x, t) \subset$ $\overline{B_{p}\left(x_{0}, r\right)}$ and hence, $T: \overline{B_{p}\left(x_{0}, r\right)} \rightarrow C B^{p}\left(\overline{B_{p}\left(x_{0}, r\right)}\right)$. Let $x \in \overline{B_{p}\left(x_{0}, r\right)}$, then we have

$$
\begin{aligned}
H_{p}\left(T\left(x, t_{0}\right), T\left(x_{0}, t_{0}\right)\right) & \leqslant a p\left(x, x_{0}\right)+b p\left(x, T\left(x, t_{0}\right)\right)+c p\left(x_{0}, T\left(x_{0}, t_{0}\right)\right) \\
& \leqslant a p\left(x, x_{0}\right)+b\left[p\left(x, x_{0}\right)+p\left(x_{0}, T\left(x, t_{0}\right)\right)\right]+c p\left(x_{0}, x_{0}\right) .
\end{aligned}
$$

Since $p\left(x_{0}, T\left(x, t_{0}\right)\right) \leqslant H_{p}\left(T\left(x_{0}, t_{0}\right), T\left(x, t_{0}\right)\right)$, we get

$$
H_{p}\left(T\left(x, t_{0}\right), T\left(x_{0}, t_{0}\right)\right) \leqslant(a+b) p\left(x, x_{0}\right)+b H_{p}\left(T\left(x_{0}, t_{0}\right), T\left(x, t_{0}\right)\right)
$$

and so we deduce that

$$
H_{p}\left(T\left(x, t_{0}\right), T\left(x_{0}, t_{0}\right)\right) \leqslant \frac{a+b}{1-b} p\left(x, x_{0}\right)=k p\left(x, x_{0}\right) .
$$


Suppose $y \in T(x, t)$, then using the last inequality, we obtain

$$
\begin{aligned}
p\left(y, x_{0}\right) & =p\left(y, T\left(x_{0}, t_{0}\right)\right) \leqslant H_{p}\left(T(x, t), T\left(x_{0}, t_{0}\right)\right) \\
& \leqslant H_{p}\left(T(x, t), T\left(x, t_{0}\right)\right)+H_{p}\left(T\left(x, t_{0}\right), T\left(x_{0}, t_{0}\right)\right)-\inf _{w \in T\left(x, t_{0}\right)} p(w, w) \\
& \leqslant M \eta\left(t, t_{0}\right)+k p\left(x, x_{0}\right)<M \epsilon+k r \leqslant(1-k) r+k r=r .
\end{aligned}
$$

Therefore, $y \in \overline{B_{p}\left(x_{0}, r\right)}$ and so, for each fixed $t \in\left(t_{0}-\delta, t_{0}+\delta\right)$, we have $T(x, t) \subset$ $\overline{B_{p}\left(x_{0}, r\right)}$. Thus, $T(\cdot, t): \overline{B_{p}\left(x_{0}, r\right)} \rightarrow C B^{p}\left(\overline{B_{p}\left(x_{0}, r\right)}\right)$ and $T(\cdot, t)$ satisfies all the conditions of Corollary 3 and has a fixed point in $\overline{B_{p}\left(x_{0}, r\right)} \subset F$. By (i), this fixed point must be in $U$, therefore, $\left(t_{0}-\delta, t_{0}+\delta\right) \subset Q$ and hence, $Q$ is open. Thus, $Q=[\alpha, \beta]$ and $T(\cdot, t)$ has a fixed point in $U$ for all $t \in[\alpha, \beta]$.

For uniqueness, fixed $t \in[\alpha, \beta]$, then there exists $x \in F$ such that $x \in T(x, t)$. If $y$ is another fixed point of $T(\cdot, t)$, then from (iv) we have

$$
\begin{aligned}
p(x, y) & =H_{p}(T(x, t), T(y, t)) \leqslant a p(x, y)+b p(x, T(x, t))+c p(y, T(y, t)) \\
& =a p(x, y)+b p(x, x)+c p(y, y) \leqslant(a+b+c) p(x, y)<p(x, y),
\end{aligned}
$$

which is a contradiction. Therefore, for any fixed $t \in[\alpha, \beta]$, the fixed point of $T(\cdot, t)$ is unique.

Acknowledgments. The authors gratefully acknowledge Editor and anonymous Reviewers for their carefully reading of the paper and helpful suggestions. C. Vetro is member of the Gruppo Nazionale per l'Analisi Matematica, la Probabilità e le loro Applicazioni (GNAMPA) of the Istituto Nazionale di Alta Matematica (INdAM).

\section{References}

1. M. Abbas, T. Nazir, S. Romaguera, Fixed point results for generalized cyclic contraction mappings in partial metric spaces, RACSAM, Rev. R. Acad. Cienc. Exactas Fís. Nat. Ser. A Mat., 106:287-297, 2012.

2. S.M.A. Aleomraninejad, Sh. Rezapour, N. Shahzad, On fixed point generalizations of Suzuki's method, Appl. Math. Lett., 24:1037-1040, 2011.

3. I. Altun, F. Sola, H. Simsek, Generalized contractions on partial metric spaces, Topology Appl., 157:2778-2785, 2010.

4. H. Aydi, Fixed point results for weakly contractive mappings in ordered partial metric spaces, J. Adv. Math. Stud., 4:1-12, 2011.

5. H. Aydi, Common fixed points for four maps in ordered partial metric spaces, Fasc. Math., 49:15-31, 2012.

6. H. Aydi, M. Abbas, C. Vetro, Partial Hausdorff metric and Nadler's fixed point theorem on partial metric spaces, Topology Appl., 159:3234-3242, 2012. 
7. H. Aydi, C. Vetro, W. Sintunavarat, P. Kumam, Coincidence and fixed points for contractions and cyclical contractions in partial metric spaces, Fixed Point Theory Appl., 2012(124):1-18, 2012.

8. V. Berinde, F. Vetro, Common fixed points of mappings satisfying implicit contractive conditions, Fixed Point Theory Appl., 2012(105):1-8, 2012.

9. P.Z. Daffer, H. Kaneko, Fixed points of generalized contractive multi-valued mappings, J. Math. Anal. Appl., 192:655-666, 1995.

10. C. Di Bari, P. Vetro, Fixed points for weak $\varphi$-contractions on partial metric spaces, International Journal of Engineering, Contemporary Mathematics and Sciences, 1:5-13, 2011.

11. C. Di Bari, P. Vetro, Common fixed points for $\psi$-contractions on partial metric spaces, Hacet. J. Math. Stat., 42:591-598, 2013.

12. L.S. Dube, S.P. Singh, On multivalued contraction mapping, Bull. Math. Soc. Sci. Math. Répub. Soc. Roum., Nouv. Sér. 14:307-310, 1970.

13. K. Iseki, Multivalued contraction mappings in complete metric spaces, Rend. Semin. Mat. Univ. Padova, 53:15-19, 1975.

14. S. Itoh, W. Takahashi, Single valued mappings, multivalued mappings and fixed point theorems, J. Math. Anal. Appl., 59:514-521, 1977.

15. E. Karapınar, Weak $\phi$-contraction on partial metric spaces, J. Comput. Anal. Appl., 14:206210, 2012.

16. P. Kumam, C. Vetro, F. Vetro, Fixed points for weak $\alpha-\psi$-contractions in partial metric spaces, Abstr. Appl. Anal., 2013:1-9, Article ID 986028, 2013.

17. S.G. Matthews, Partial metric topology, Ann. N. Y. Acad. Sci., 728(1):183-197, 1994.

18. S.B. Nadler, Multivalued contraction mappings, Pacific J. Math., 30:475-488, 1969.

19. D. Paesano, P. Vetro, Suzuki's type characterizations of completeness for partial metric spaces and fixed points for partially ordered metric spaces, Topology Appl., 159:911-920, 2012.

20. A. Petruşel, Integral inclusions. Fixed point approaches, Pr. Mat., 40:147-158, 2000.

21. B.K. Ray, On Ćirić's fixed point theorem, Fundam. Math., 94:221-229, 1977.

22. S. Romaguera, On Nadler's fixed point theorem for partial metric spaces, Math. Sci. Appl. E-Notes, 1:1-8, 2013.

23. M. Sgroi, C. Vetro, Multi-valued $F$-contractions and the solution of certain functional and integral equations, Filomat, 27:1259-1268, 2013.

24. S. Shukla, S. Radenović, C. Vetro, Set-valued Hardy-Rogers type contraction in 0-complete partial metric spaces, Int. J. Math. Math. Sci., 2014:1-9, Article ID 652925, 2014.

25. F. Vetro, S. Radenović, Nonlinear $\psi$-quasi-contractions of Ćirić-type in partial metric spaces, Appl. Math. Comput., 219:1594-1600, 2012.

26. H. Yingtaweesittikul, Suzuki type fixed point theorems for generalized multi-valued mappings in b-metric spaces, Fixed Point Theory Appl., 2013(215):1-9, 2013. 